\title{
Parkinson-Like Symptom as a Rare Manifestation of Systemic Lupus Erythematosus: A Case Report
}

\author{
Razzaque $\mathrm{MA}^{1}$, Haider $\mathrm{MZ}^{2}$, ${ }^{*}$ Sazzad $\mathrm{MN}^{3}$, Ahmed $\mathrm{S}^{4}$, Shahin $\mathrm{MA}^{5}$, Haq $\mathrm{SA}^{6}$
}

\begin{abstract}
A 65-year old Bangladeshi woman with Systemic Lupus Erythematosus (SLE) developed Parkinson-like movement disorder. Steroid pulse therapy followed by prednisolone was most effective in this case. Psychosis, seizure and meningitis are common central nervous system (CNS) manifestations in SLE patients, and Parkinson-like rigidity or tremors are rare.
\end{abstract}

Keywords: Systemic Lupus Erythematosus, CNS lupus, Parkinson-like rigidity, Steroid pulse therapy.

\section{INTRODUCTION}

Patients with SLE, CNS involvement may have varied presentations like headache, cognitive dysfunction, seizure, cerebrovascular disease, acute confusional state and psychiatric disturbance. Movement disorders like chorea, hemiballismus, cerebral ataxia and Parkinson-like rigidity or tremors are rare manifestations ${ }^{1}$. We came across a woman with SLE and Parkinson-like symptoms, an extremely rare form of CNS lupus manifestation.

\section{CASE REPORT}

A 65-year old Bangladeshi woman with Diabetes Mellitus (DM) and Hypothyroidism was diagnosed as a case of SLE on the basis of polyarthritis, photosensitive malar rash, and alopecia, positive anti Nuclear Antibody (ANA) and anti ds DNA in 2009. She was treated with hydroxyl chloroquine

1. Dr. Md. Abdur Razzaque, Resident, Phase B, Dept of Rheumatology, Bhangabandhu Sheikh Mujib Medical University (BSMMU), Shahbag, Dhaka

2. Dr. Mohammad Ziaul Haider, Resident, Phase B, Dept of Rheumatology, BSMMU.

3. *Dr. Md. Nahduzzamane Sazzad, Medical Officer, Dept of Rheumatology. BSMMU.

Email: shazzad8@gmail.com

4. Dr. Shamim Ahmed, Associate Professor, Dept of Rheumatology. BSMMU.

5. Dr. Md. Abu Shahin, Associate Professor, Dept of Rheumatology. BSMMU.

6. Dr. Syed Atiqul Haq. Professor, Dept of Rheumatology. BSMMU.

${ }^{*}$ For correspondence
(HCQ), methotrexate (MTX) and prednisolone on a tapering course along with thyroxin. She improved with these medications. Later MTX was stopped due to haemolysis and azathioprine was added. At the same time, course of prednisolone was completed but the patient developed Jaccoud's like deformities in metacarpophalangeal joints of right hand and interphalangeal joints of right foot. She also developed Coombs' positive autoimmune haemolytic anemia. Subsequently she developed difficulty in walking, slowness of speech with behavioural alteration (like apathy, mutism and irritability), bradykinesia and rigidity. She was considered as a case of Parkinson Disease (PD) and put on Levodopa $150 \mathrm{mg}$ along with Carbidopa $15 \mathrm{mg}$ daily for four years. There was gradual progression of symptoms, there by Ropinirole was added but no improvement was seen. She had a single episode of convulsion in 2011. In 2012 she experienced low trauma fracture of neck of left femur and pulmonary embolism. She had no history of unconsciousness, nasal regurgitation, swallowing difficulties and bowelbladder abnormalities.

She had mild splenomegaly $(12.4 \mathrm{~cm})$, no ascites, GIT bleeding or sign of CLD. There were no abnormalities detected in heart and lungs. Her consciousness was clear. She had expressionless face with slow and monotonous speech. There was cogwheel and lead pipe rigidity of upper and lower limbs. Muscle power was 4/5 in both proximal and distal groups with diminished deep reflexes, equivocal plantar reflexes bilaterally with short step gait.

Laboratory examination showed haemoglobin $5.3 \mathrm{gm} / \mathrm{dl}$, CRP was 6, ANA and anti ds DNA were positive, liver function, renal function and urine $\mathrm{R} / \mathrm{E}$ were within normal limits. Anti carddiolipinAb positive but Lupus anti-coagulant, $\beta_{2}$ Glycoprotein-1 $\operatorname{IgM}$ and $\operatorname{IgG}$ were negative. Vitamin D total was $32.1 \mathrm{ng} / \mathrm{ml}$. Rheumatoid factor, anti CCP, anti SmAb, anti SSA, Anti SSB, HBs Ag, anti HCV, ICT for Malaria and Kala azar, anti Mitochondrial Ab, Anti LKM Ab were negative. CT scan and MRI of the brain showed age related mild cortical atrophy and brain SPECT revealed hypo perfusion of fronto-parietal region and left basal ganglion. 


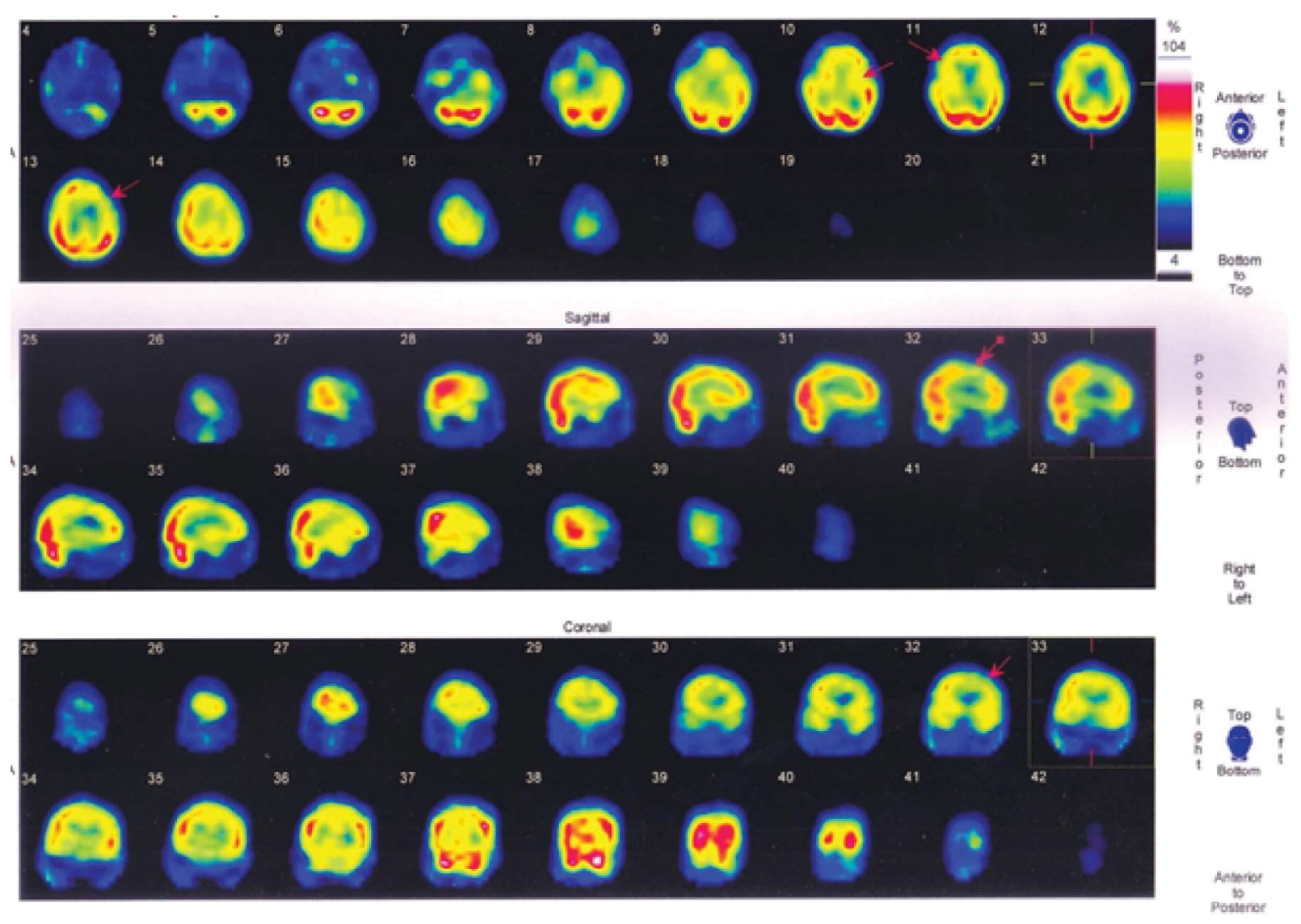

Fig 1: SPECT showinghypoperfusion of fronto-parietal regeion of both cortex(Right $>$ Left) and basal ganlia(Left)

Diagnosis of CNS lupus with Parkinsonian-like symptoms was added, stopped all anti Parkinson drugs and steroid pulse therapy $(800 \mathrm{mg}$ Methylprednisolone daily for 3 days) was given, followed by $40 \mathrm{mg}$ daily oral prednisolone. After steroid therapy along with pulse cyclophosphamide, there were improvement of rigidity, facies, Speech, walking and reduced aggressiveness of behavior. Finally she was discharged after one month of hospital course.

\section{DISCUSSION}

Our patient was initially diagnosed as a case of SLE on the basis of polyarthritis, photosensitive malar rash, alopecia, positive ANA and anti- ds DNA in 2009. Later she developed seizure, Jaccoud'slike joint deformities, rigidity, bradykinesia, expressionless facial features, slowness of speech with behavioral alteration (as irritability, aggressiveness, apathy, mutism). Other causes of secondary Parkinsonism was not considered as the patient had never been given responsible drugs and never exposed to toxic agent like CO or Manganese. Cranial CT and MRI scan showed only degenerative changes. Brain SPECT shows hypo perfusion of fronto-parietal region and left basal ganglia. Anti- phospholipid antibodies like Anticardiolipin (aCL) Ab, lupus anti-Coagulant (LA) and $\beta_{2}$ glycoprotein-1 IgM were positive. Therefore, in this case, Parkinsonian like symptoms were considered to be manifestations of CNS lupus ${ }^{1}$. After a high dose of methyl prednisolone pulse therapy,Parkinsonian-like symptoms were improved dramatically and all anti Parkinsonian drugs were withdrawn.

Seizures, mental disorders and cranial neuropathy are the more commonly observed symptoms of CNS lupus 2,3 . Chorea is also common as extrapyramidal involvement of $\mathrm{SLE}^{4,5}$. In contrast, Parkinsonian-like symptoms are extremely rare ${ }^{6}$. Willoughby et $\mathrm{al}^{7}$ described a 30 -year-old male SLE patient with cogwheel rigidity. Despite $40 \mathrm{mg}$ daily of prednisolone, complications due to meningitis and endocarditis followed and he died. Autopsy revealed multiple areas of encphalomalacia in the basal ganglia of the brain. In a study Yancey et $\mathrm{al}^{8}, 2$ out of 37 children with SLE had Parkinsonian-like symptoms and in one child had cogwheel rigidity. The clinical outcome was not 
described. In another case, Parkinsonian-like symptoms and coma was developed in a 16-year-old girl; a nearly complete recovery was achieved. Although the authors did not describe how they had treated that particular patient, they favoured combination of high dose prednisolone $(2 \mathrm{mg} / \mathrm{kg} /$ day $)$ and a cytotoxic agent as initial therapy of CNS lupus, and steroid pulse therapy for non responding patients. Nagaokaet at. ${ }^{9}$ reported SLE in a 35-year-oldwoman with akinesia, muscle rigidity and expressionless facial features. Her Parkinsonian-like symptoms were controlled by $40 \mathrm{mg}$ daily methylpre- dnisolone and anti-Parkinsonian drugs. For this patient, antiParkinsonian drugs were necessary since symptoms relapsed during the course of steroid-tapering when amantadine was discontinued. In our patient the Parkinsonian-like symptoms were improved dramatically after Methylprednisolone pulse therapy and the patient remained in clinical remission after discharge. In many cases, CNS lupus can be effectively treated with high dose corticosteroids including methylprednisolone pulse therapy ${ }^{10}$. About $25 \%$ of CNS lupus episodes are not responsive to steroid ${ }^{11}$.In such cases, cyclophosphamide pulse therapy ${ }^{12}$ and plasma exchange ${ }^{13}$ may be effective.

Although the putative pathogenic mechanism of CNS lupus includes vasculitis in the $\mathrm{CNS}^{14}$, functional disorder of nerve cells due to auto-antibodies against neuronal cells (eg. Anti-Asialo GMI Antibody ${ }^{15}$, Anti-Ribosomal P protein Antibody ${ }^{16}$, Anti-Neuronal cell Antibody ${ }^{17}$ ), and thrombi formation in the CNS by Anti-Phospolipid Antibodies ${ }^{18}$, precise mechanisms are yet to be defined.

\section{CONCLUSIONS}

We reported a rare case of SLE, in which Parkinsonian-like symptoms were noticed as manifestation of CNS lupus. The patient's symptoms were effectively managed with high dose prednisolone with anti Parkinsonian drugs ropinirole only.

\section{REFERENCES}

1. Daniel JW, Berva HH. Dubois' Lupus Erythematosus and Related Syndromes, eight edition 2013; 29: 371-2.

2. Johnson RT, Richardson EP. The neurological manifestations of systemic lupus erythematosus. A clinical-pathological study of 24 cases and review of literature. Medicine 1968; 47: 337-69.
3. Bennahum DA, Messenar RP. Recent observation on central nervous system lupserythematosus. Semin Arthritis Rheum 1975; 4: 253-66.

4. Asherson RA, Harris EN,Gharavi AE, Hughes GRV. Systemic lupus erythematosus, antiphospholipid antibodies, chorea and oral contraceptives. Arthritis Rheum 1987; 30: 1535-6.

5. Lusuns JO, Szilagyi PA. Clinical features of chorea associated with systemic lupus erythematosus. Am J Med 1975; 58: 875-61.

6. Daniel JW, Berva HH. Dubois' Lupus Erythematosus and Related Syndromes, eight edition 2013; 29: $371-2$.

7. Willoughby EO, Cardon L, Lubnitz ME. Clinicopathological conference: psychotic episodes, meningitis and chest pain. Postgrad Med 1964; 35: 318-26.

8. Yancey CL, Doughty RD, Athereya BH. Central nervous system involvement in childhood systemic lupus erythematosus. Arthritis Rheum 1981; 12: 3849-9:5.

9. Nagaoka S, Kato K, Ishigatsubo $\mathrm{Y}$ et al. A case of systemic lupus erythematosus with akinesia, muscle rigidity and neurogenic bladder. The Rheumanchi 1984; 24: 382-8 (in Japanese).

10. Miyoshi Y, Atsumi T, Kitagawa H, Ogura N, Amasaki Y. Parkinson-like symptoms as a manifestation of systemic lupus erythematosus. Lupus 1993-lup subgroup com.

11. Wallace DJ. In Dubois' Lupus Erythematosus, 4th edition. London: Lea \&Febiger, 1993: 384-5.

12. Boumpas DT, Yamada H, Patronas NJ, Scott D, Klippel JH, Balow JE. Intemittent pulse cyclophosphamide for the treatment of severe neuropsychiatric lupus. Arthritis Rheum 1990; 33(Suppl): S103 (abstract).

13. Tanter Y, Rifle G, Chalopin JM, Mousson C, Besancenot JF. Plasma exchange in central nervous system involvement of systemic lupus erythematosus. Plasma TherTransfusTechnol 1987; 8: 161-8.

14. Harris EN, HughesGRV. Cerebral disease in systemic lupus erythematosus. Springer SeminImmunopathol 1985; 8: 251-66. 
15. Hirano $T$, Hashimoto $H$, Shiokawa $Y$ et al. Antiglycolipid autoantibody detected in the sera from systemic lupus erythematosus patient. J Clin Invest 1980; 66:1437-40.

16. GolombeckSJ,Graus F, Elkon KB. Autoantibodies in the cerebrospinal fluid of patients with systemic lupus erythematosus. Arthritis Rheum 1986; 29: 1090-7.
17. Bluestein HG, Williams GW,Steinberg AD. Cerebrospinal fluid antibodies to neuronal cells: associated with neuropsychiatric manifestations of systemic lupus erythematosus. Am J Med 1981; 70: 240-6.

18. Harris EN, Gharavi AE, Asherson RA, Boey MI, Hughes GR. Cerebral infarct in systemic lupus erythematosus associated with anticardiolipin antibodies. ClinExpRheumatol 1984; 2: 47-51. 